\title{
Free LIF receptor $\alpha$-chain distal cytoplasmic motifs enhance Jak2-independent STAT3 phosphorylation and induce differentiation in HL-60 cells
}

\author{
QING SUN ${ }^{1,2^{*}}$, JING WANG $^{1 *}$, JUN XIONG $^{1}$, LING YANG $^{1}$ and HOUQI LIU ${ }^{1}$ \\ ${ }^{1}$ Department of Embryology and Histology, Second Military Medical University, \\ Shanghai 200433; ${ }^{2}$ No. 401 Hospital of PLA, Qingdao 266071, P.R. China
}

Received February 22, 2011; Accepted March 21, 2011

DOI: 10.3892/or.2011.1289

\begin{abstract}
The leukemia inhibitory factor (LIF) affects multiple types of leukemia cells in vitro through the functional LIF receptor (LIFR), which comprises a complex of the LIFR $\alpha$-chain (LIFR $\alpha$ ) and gp130. As Jak2/STAT3 has been proven to be a significant mediator in the LIF-induced differentiation of promyeloid leukemia cells, we constructed a recombinant vector, pcDNA3.0-CT3 (containing the structurally conserved triple YXXQ motifs of LIFR $\alpha$, termed LIFR $\alpha$-CT3), and its specific tyrosine-mutated counterpart, pcDNA3.0-MUT, to determine the sites and examine the corresponding mechanisms involved in STAT3 phosphorylation. We found that the triple YXXQ motifs of LIFR $\alpha$-CT3 are capable of up-regulating phosphorylated levels of STAT3 in a Jak2-independent manner prior to the induction of myeloid differentiation by LIFR $\alpha$-CT3 in the human promyeloid cell line HL-60. By specifically blocking Jak2 using the AG-490 inhibitor, we observed that the LIFR $\alpha$-CT3 group of HL-60 cells still demonstrated up-regulation of phosphorylated STAT3 and this up-regulation could result in the myeloid differentiation of HL-60 cells. These results may shed light on acute promyeloid leukemia therapy in future clinical practice.
\end{abstract}

\section{Introduction}

The leukemia inhibitory factor (LIF) has been cloned and purified on the basis of its capacity to induce differentiation of the M1 murine myeloid leukemia cell line $(1,2)$. As a member

Correspondence to: Professor Houqi Liu, Department of Embryology and Histology, Second Military Medical University, Shanghai 200433, P.R. China

E-mail: houqiliu@gmail.com

*Contributed equally

Key words: acute promyeloid leukemia, Jak/STAT pathway, leukemia inhibitory factor receptor, leukemia inhibitory factor receptor, gp130, IL-6 of the IL-6 family of cytokines, LIF mediates its biological effects by binding to its receptor, which consists of a lowaffinity LIFR $\alpha$-chain termed LIFR $\alpha$ and a high-affinity subunit termed gp130, both of which are found in other IL-6 family members (3). These cytokines thus initiate intracellular signaling by triggering LIFR $\alpha$-gp130 heterodimerization or gp130-gp130/LIFR $\alpha$-LIFR $\alpha$ homodimerization via the Janus kinase/signal transducer and activator of transcription (Jak/STAT) pathway. In the human promyeloid cell line HL-60, this signaling process is primarily mediated by the Jak2/STAT3 pathway (4).

STAT3 has been shown to play a central role in LIF-induced differentiation in leukemia cells (5). Generally speaking, after heterodimerization or homodimerization mediates the activation of intracellular Jak2, Jak2 phosphorylates six tyrosine residues (Y) on the intracellular C-terminus of gp130. Whereas, the second membrane-distal tyrosine residue (Y2) is required for recruitment of the $\mathrm{SH} 2$-containing phosphatase-2, any one of the four tyrosine residues (Y3-Y6) containing the YXXQ motif can serve as a docking site for STAT3 binding (6). STAT3 is subsequently phosphorylated by Jak2 on Y705 on the C-terminus, which leads to the formation of STAT3 dimers via reciprocal interactions of the $\mathrm{SH} 2$ domain and the phosphorylated Y705. Upon dimerization, STAT3 translocates into the nucleus, binds to DNA and regulates target genes leading to various cellular responses (7). While this is the canonical pathway for STAT3 activation, there have been reports claiming that STAT3 could be activated by other molecules, suggesting that the key structure in STAT3 activation is not gp130-specific, but is located on LIFR $\alpha$ (8). Intriguingly, studies have shown that the intracellular amino acid 136-145 region of the distal $\mathrm{C}$-terminus contains five tyrosine residues (Y5) and several YXXQ motifs that are genetically conserved among gp130, LIFR $\alpha$ and G-CSFR $(9,10)$. Thus, it is possible that free LIFR $\alpha$-CT3 in the cytoplasm could also enhance or even initiate, STAT3 phosphorylation, thus triggering downstream signaling.

In our present study, we demonstrate that intracellular enrichment of LIFR $\alpha$-CT3 in human promyeloid HL-60 cells enhances STAT3 phosphorylation in a Jak2-independent manner through specific triple $\mathrm{YXXQ}$ motifs located on the distal C-terminus. These results confirm the bioactivity of 
phosphorylated STAT3 (pSTAT3) in the induction of HL-60 myeloid differentiation.

\section{Materials and methods}

Cell culture, antibodies and inhibitors. The human promyeloid leukemia cell line HL-60 was purchased from Cell Bank, Chinese Academy of Sciences (Shanghai, China), and cultured as described previously (11). Antibodies used for immunoblotting were as follows: primary antibodies against Jak2, phosphorylated Jak2 (pJak2), total STAT3 (tSTAT3), phosphorylated STAT3 Y705 (pSTAT3) and $\beta$-actin and were all purchased from Santa Cruz Biotechnology. FITC-conjugated goat anti-mouse antibody (green) and 4'-6-diamidino-2-phenylindole (DAPI, blue) were purchased from KangChen (Shanghai, China). Recombinant human LIF (hLIF) was purchased from Chemicon (Temecula, CA).

A STAT3-specific peptide inhibitor that functions as a highly selective, potent blocker of STAT3 phosphorylation (No. 573096) and a Jak2-specific inhibitor (AG-490) were both purchased from Calbiochem (La Jolla, CA).

RT-PCR, vector construction and transfection. Total RNA extraction and RT-PCR were performed as described by Fang et al (12) with the following primers (synthesized by Invitrogen, Shanghai, China): LIFR $\alpha$-CT3: 5'-TAGGATCC GTCGATGTATCAGCCTCAAGCA-3' (forward) and 5'-GG TCTAGAAGTGACACGGTGACACTGTTAA-3' (reverse). The forward and reverse primers contained $\mathrm{BamHI}$ and $\mathrm{XbaI}$ sites, respectively, as underlined. The amplified fragments were cleaved by BamHI and $\mathrm{XbaI}$ and then ligated into a pcDNA3.0 vector (Invitrogen, Carlsbad, CA) that had been previously

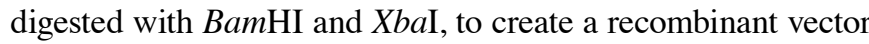
containing LIFR $\alpha$-CT3 termed pcDNA3.0-CT3. The inserted genes were verified by DNA sequencing (Invitrogen).

For the recombinant vector pcDNA3.0-MUT, overlapping extension PCR (OE-PCR) was performed to mutate the leading amino acid residue $\mathrm{Y}$ (tyrosine) in all three $\mathrm{YXXQ}$ motifs contained in LIFR $\alpha$-CT3 to F (phenylalanine) in order to eliminate any phosphorylation at these sites. Three pairs of primers were synthesized by Invitrogen as follows: Plu-1: 5'-GGGGGGATCCTCGATGTTCCAGCC-3' (forward), Pld-1: 5'-GAGGTGCATCTGTGGCTTGAAGCC-3' (reverse); Pup-2: 5'-AAGCCACAGATGCACCTCCC-3' (forward), Pld-2: 5'-ATTGGCCTGAGGTCTGAAACC-3' (reverse); Plu-3: 5'-AGACCTCAGGCCAATGTAAA-3' (forward), Pld-3: 5'-GGGGTCTAGAGGTCTAGAAGTGAC-3' (reverse). The PCRs were carried out with $P f u$ DNA polymerase according to the manufacturer's protocol (Fermentas, Hanover, MD). A 35-cycle PCR with standard procedures was carried out to ligate the three amplified fragments together before being verified by DNA sequencing. The end product, having a size of $\sim 600$ base pairs (bp), was then cleaved with BamHI and $\mathrm{Xba \textrm {I }}$ (as underlined) to construct pcDNA3.0-MUT using the same protocol as that used for pcDNA3.0-CT3.

Three stable HL-60 cell lines were established using Fugene 6-mediated transfection (Roche, Basel, Switzerland). G418 selection $(200 \mu \mathrm{g} / \mathrm{ml})$, expressing pcDNA3.0-CT3 (CT3), pcDNA3.0-MUT (MUT) and a blank vector pcDNA3.0 (3.0) G418-resistant clones were then isolated and expanded.
Western blot analysis. The CT3, MUT and wild-type (WT) HL-60 cell lines were prepared by lysing cells at $1 \times 10^{7}$ cells $/ \mathrm{ml}$, rinsing with ice-cold $1 \mathrm{X}$ PBS for 3 times and extracting in RIPA buffer containing $1 \mathrm{mM}$ sodium fluoride, $2 \mathrm{mM}$ sodium orthovanadate and a cocktail of protease inhibitors (Santa Cruz Biotechnology, Santa Cruz, CA). Immunoblotting was then carried out using a Tricine-SDS-PAGE gel as described previously (13). For normalization of protein loading, the same membranes were processed using the same procedure with an antibody against intracellular tSTAT3, Jak 2 or $\beta$-actin as an internal control. The strip amounts were quantified by densitometry scanning using a GS-700 densitometer (Bio-Rad Laboratories, Inc., CA, USA) and were analyzed with Quantity One 4.5.2 software (Bio-Rad Laboratories, Inc.).

Flow cytometry analysis. Two differential markers of myeloid leukemia, CD11b and CD14, were measured by flow cytometry (Becton-Dickinson, Franklin Lakes, NJ, USA) as previously described (14). In brief, cells were collected, rinsed, and incubated with a monoclonal mouse anti-human PE-conjugated anti-CD11b antibody and a mouse anti-human FITC-conjugated anti-CD14 antibody for $30 \mathrm{~min}$ at $37^{\circ} \mathrm{C}$. The fluorescence intensity was analyzed by flow cytometry. Data were based on the examination of $1.0 \times 10^{5}$ cells/sample randomly selected from $\sim 5 \times 10^{5}$ cells. The results were analyzed using FACSCalibur with CellQuest software version 3.0 (Becton-Dickinson, San Jose, CA, USA).

Immunofluorescence assay. The pre-staining treatment of non-adherent HL-60 cells $\left(\sim 1 \times 10^{6}\right.$ cells $\left./ \mathrm{ml}\right)$ was performed as previously described (15). The smears were incubated with a mouse anti-human pSTAT3 primary antibody diluted in blocking serum (1:50), incubated for $30 \mathrm{~min}$ at $37^{\circ} \mathrm{C}$ and then rinsed three times for $5 \mathrm{~min}$ each in $4 \mathrm{X} \mathrm{SSC} / 0.1 \%$ Tween-20. The blocking step was repeated before smears were incubated with a FITC-conjugated secondary antibody diluted in blocking solution (1:200) and subsequently rinsed as previously described. The smears were mounted in antifade solution for analysis. The nuclei of the HL-60 cells were counterstained with DAPI, visualized by a fluorescence microscope (Leica, Wetzlar, Germany) with a x630 magnification and were photographed using a charge-coupled camera.

Statistical analysis. All studies were performed a minimum of three times, and the data are expressed as the mean \pm SEM. The results were considered statistically significant if the p-value was $<0.05$ as determined by one-way ANOVA or t-tests.

\section{Results}

The triple YXXQ motifs of LIFR $\alpha$-CT3 are overexpressed in $H L-60$ cells. The full-length LIFR $\alpha$-CT3 consists of 118 amino acid residues. To produce an overexpression model for LIFR $\alpha$-CT3 in HL-60 cells, an expression vector, pcDNA3.0CT3 (CT3), was constructed. A recombinant vector with the $\mathrm{Y}$ residue in all three $\mathrm{YXXQ}$ motifs contained in LIFR $\alpha$-CT3 mutated to F (MUT) was also constructed as the control (Fig. 1A). After stable transfection and G418 selection, the protein levels of the WT, 3.0, MUT and CT3 groups were 


\section{$\mathbf{A}$}

Score $=615$ bits $(320)$, Expect $=2 e-173$
Identities $=346 / 349(99 \%)$, Gaps $=$

St $r$ and $=$ Plus/Plus

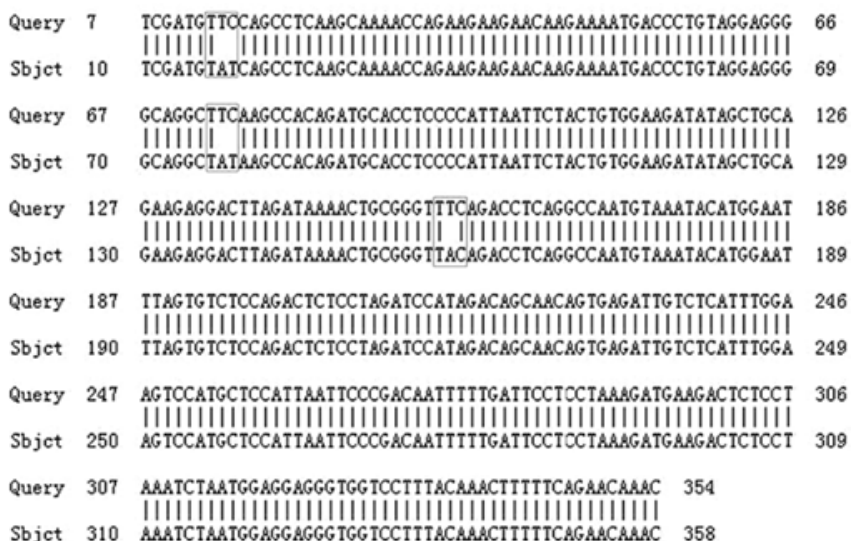

$\begin{array}{lllll}\text { B } & \text { WT } & 3.0 & \text { MUT } & \text { CT3 }\end{array}$
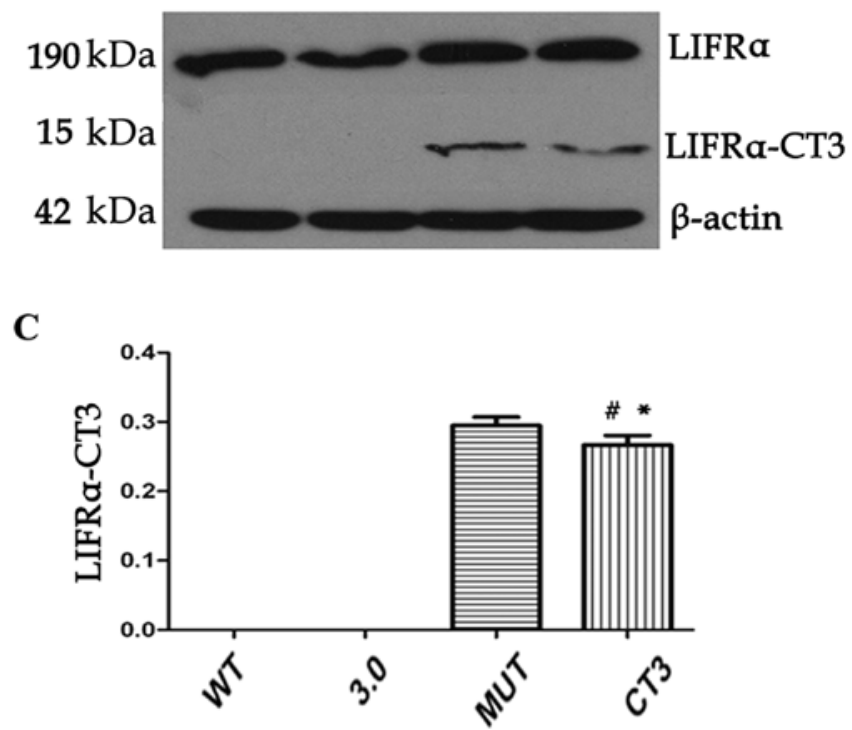

Figure 1. Overexpression of LIFR $\alpha$-CT3 in HL-60 cells. (A) Nucleotide comparison between the mutated and the original LIFR $\alpha$-CT3 sequence on the triple $\mathrm{YXXQ}$ motifs using the Basic Local Alignment Search Tool (BLAST, http://www.ncbi.nlm.nih.gov/blast). The three mutated sites are highlighted. (B and C) Western blot analysis and corresponding densitometry analysis (C) were carried out on the WT (wild-type HL-60 cells), 3.0 (stably transfected with the blank pcDNA3.0), MUT (stably transfected with pcDNA3.0-MUT) and CT3 (stably transfected with pcDNA3.0-CT3) groups using an antibody against the LIFR C-terminus (1:1000). $\beta$-actin is shown as an internal reference. Values are the means \pm SEM ( $n=3$ for each group). " $\mathrm{p}<0.05$ when compared to the 3.0 group. ${ }^{*} \mathrm{p}<0.05$ when compared to the WT.

determined by immunoblotting (Fig. 1B). When probed with the LIFR C-terminal antibody, both the MUT and CT3 groups showed a LIFR $\alpha$-CT3-specific protein band $(\sim 15 \mathrm{kDa})$ in addition to the endogenous LIFR $\alpha(\sim 190 \mathrm{kDa})$. For the MUT group, the appearance of a $\sim 15 \mathrm{kDa}$ band could be reasonably attributed to intact LIFR C-terminal antibody binding sites, despite the presence of three amino acid mutations. The 3.0 and WT groups, however, exhibited a single endogenous protein band ( $15 \mathrm{kDa})$. Densitometry analysis of the immunoblotting results (Fig. 1C) confirmed that the expression of the $15-\mathrm{kDa}$ protein in the CT3 group was significantly higher than
A

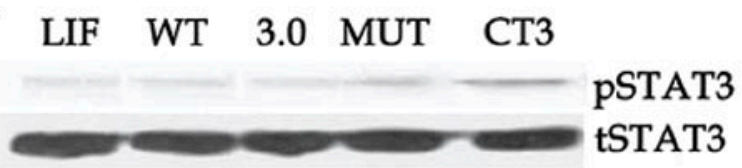

\section{B}

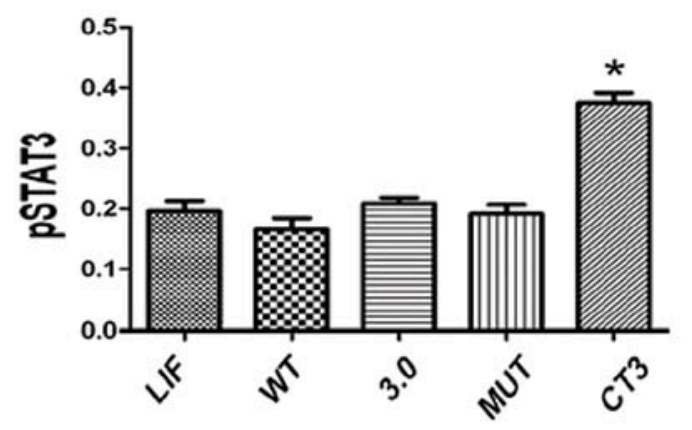

C

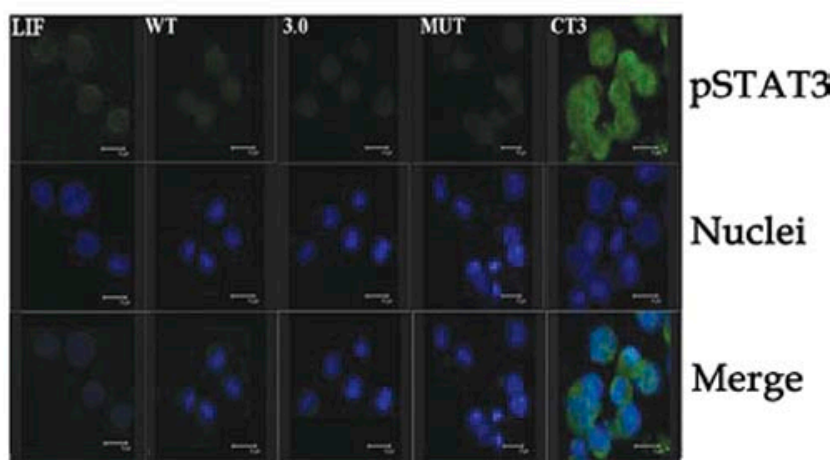

Figure 2. Intracellular LIFR $\alpha$-CT3 enrichment enhances STAT3 phosphorylation in HL-60 cells. (A and B) HL-60 cells were incubated in serum-free medium for $5 \mathrm{~h}$ and treated with hLIF $(100 \mathrm{ng} / \mathrm{ml})$ for $30 \mathrm{~min}$ prior to being prepared as described (13). Western blot analysis (A) and corresponding densitometry analysis (B) were carried out using an antibody against Y705 phospho-STAT3 (pSTAT3) and re-stained with an antibody against total STAT3 (tSTAT3) as an internal control. Values are the means \pm SEM $(n=3$ for each group). ${ }^{*} \mathrm{p}<0.05$ when compared with the other groups. (C) The intracellular distribution of pSTAT3 among the HL-60 cell groups is shown using immunofluorescence microscopy. The CT3 group exhibited a greater fluorescence intensity in the nucleus when labeled with an antibody specific for Y705 pSTAT3 as compared to the other groups. DAPI was chosen to counterstain the nuclei of the HL-60 cells. Magnification, x630.

that in the $3.0(\mathrm{p}<0.05)$ and the WT $(\mathrm{p}<0.05)$ groups. The data provide evidence that LIFR $\alpha$-CT3 is overexpressed in HL-60 cells.

The triple YXXQ motifs of LIFR $\alpha$-CT3 enhance STAT3 phosphorylation. Our laboratory previously reported that homodimerization of the entire LIFR $\alpha$ cytoplasmic region can promote Y705 phosphorylation and inhibit the proliferation of HL-60 cells (13). To explore the phosphorylating sites and protein level changes caused by LIFR $\alpha$, we compared the levels of pSTAT3 using a phospho-specific Y705 antibody in the CT3, WT, 3.0 and MUT groups in addition to cells that were directly stimulated with LIF in vitro at a final concentration of $100 \mathrm{ng} / \mathrm{ml}$ (Fig. 2A). tSTAT3 and pSTAT3 were detected in all protein lysates, which is consistent with a 
A

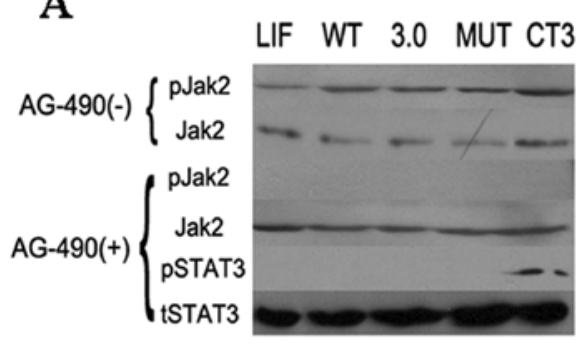

B
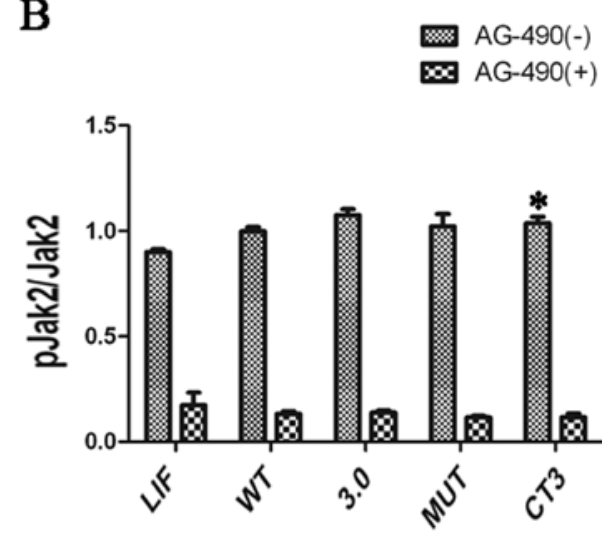

C

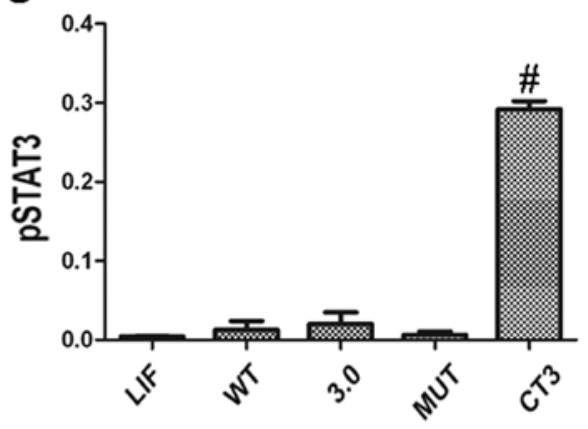

Figure 3. Up-regulation of pSTAT3 in LIFR $\alpha$-CT3 ${ }^{+}$HL-60 cells is Jak2independent. (A) The WT, 3.0, MUT and CT3 groups were starved in serum-free medium prior to addition of the Jak2-specific inhibitor, AG-490 $(100 \mu \mathrm{M})$, for $24 \mathrm{~h}$ and then harvested for Western blot analysis $(18,30,31)$. For the LIF group, AG-490 was added to starving HL-60 cells in serum-free medium $(100 \mu \mathrm{M})$ for $24 \mathrm{~h}$ and rinsed three times with ice-cold 1X PBS before treatment with hLIF $(100 \mathrm{ng} / \mathrm{ml})$ for $30 \mathrm{~min}$ prior to processing. The pJak2 and pSTAT3 Y705 levels in the total cell lysates were determined by Western blot analysis. Jak2 and tSTAT3 were used as loading controls, respectively. Values are the means \pm SEM ( $n=3$ for each group). (B) The efficacy of AG-490 $(100 \mu \mathrm{M}, 24 \mathrm{~h})$ in inhibiting pJak2 was significant (>85\%); * $\mathrm{p}<0.05$ when the AG-490(+) sample is compared with the AG-490(-) control for each of the groups. (C) Following the treatment of AG-490 $(100 \mu \mathrm{M}$, $24 \mathrm{~h}$ ), densitometry analysis was applied to evaluate its effect on endogenous pSTAT3. ${ }^{*} \mathrm{p}<0.05$ when the CT3 group is compared with the other groups.

previous report confirming that STAT is consecutively activated among hemopoietic tumors (16). However, only the CT3 group exhibited a significantly higher level of pSTAT3. In particular, the LIF group showed no observable pSTAT3 up-regulation in the HL-60 cells, which agrees with the well-established theory that, when acting alone, LIF has no observable effects on the number, size or morphology of colonies formed by HL-60 (17).

Results from immunofluorescence microscopy analysis show the intracellular pSTAT3 distribution among the HL-60 groups (Fig. 2C). Intracellular pSTAT3 was up-regulated in the $\mathrm{CT} 3$ group but not in the other groups.
STAT3 phosphorylation in LIFR $\alpha-C T 3^{+} H L-60$ cells is Jak2-independent. To provide additional evidence that free LIFR $\alpha$-CT3 in the cytoplasm is responsible for STAT3 activation, a Jak2-specific inhibitor, tyrphostin AG-490, was used to block endogenous STAT3 Y705 phosphorylation (18). All groups of HL-60 cells were treated as described in Materials and methods and subsequently processed for Western blot analysis (Fig. 3A). Densitometry analysis confirmed that the levels of pSTAT3 were uniquely elevated in the CT3 group compared to the other groups, even after blocking of endogenous pSTAT3 mediated by pJak2 (>85\%) in all HL-60 cells (Fig. 3B and C). These results suggest that LIFR $\alpha$-CT3 is responsible for enhanced pSTAT3 levels in HL-60 cells independently of Jak2 and that this specific effect is stronger than when hLIF is directly administered to leukemia cells in vitro.

LIFR $\alpha$-CT3-induced up-regulation of pSTAT3 promotes differentiation of $\mathrm{HL}-60$ cells. To further delineate the effects of LIFR $\alpha$-CT3-related pSTAT3 up-regulation on HL-60 cell differentiation, we analyzed all five groups of HL-60 cells using FACSCalibur with the CellQuest program to quantify the levels of surface promyeloid differentiation markers, such as CD14 and CD11b (Fig. 4A). The results suggest that the myeloid lineage of the CT3 group was more differentiated than that of the other four groups due to the statistically higher expression of both CD14 and CD11b.

To determine the role of STAT3 in promyeloid leukemia, we used a cell-permeable STAT3-specific inhibitor at the recommended dosage $(25 \mu \mathrm{M})(19)$. As shown in Fig. 4B-D, while the inhibition of pSTAT3 was highly efficient among the groups (>85\%, Fig. $4 \mathrm{C}$ and D), the CT3 group exhibited a restoration of malignancy, as represented by the increased expression of CD11b and CD14 compared to the same groups that were not administrated with STAT3-inhibitor. These results suggest that the higher level of pSTAT3 is pivotal in the CT3 group and is closely associated with myeloid leukemia differentiation. The pSTAT3 intracellularly activated by LIFR $\alpha$-CT3 independently of Jak2 is biologically active in the induction of leukemia cell differentiation.

\section{Discussion}

In the present study, we identified the phosphorylating sites of LIFR $\alpha$-CT3 and demonstrated that its intracellular enrichment by transfection could activate Jak2-independent STAT3 phosphorylation and induce myeloid differentiation of HL-60 cells.

Several studies have shown that constitutive STAT3 activation is inevitable in gp130-mediated myeloid differentiation or in growth arrest of M1 leukemia cells induced by LIF/IL-6 (20-22). However, Tomida et al demonstrated that the YXXQ motifs of LIFR, which are homologous to those present in gp130, could likewise generate signals for growth arrest and differentiation of myeloid leukemia cells (23). Unlike the toxic effects of LIF on leukemia cells when directly applied (24), our data show that overexpression of LIFR $\alpha$-CT3 in the cytoplasm could result in LIF-like differentiation effects on HL-60 cells.

pSTAT3 up-regulation has been associated with pathological lesions in myeloid leukemia patients and recent studies 
A

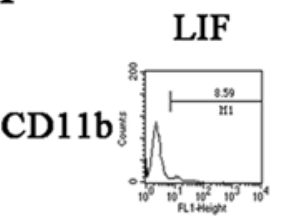
WT 3.0

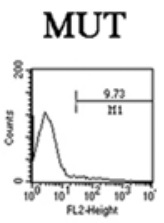
CT3

CD14
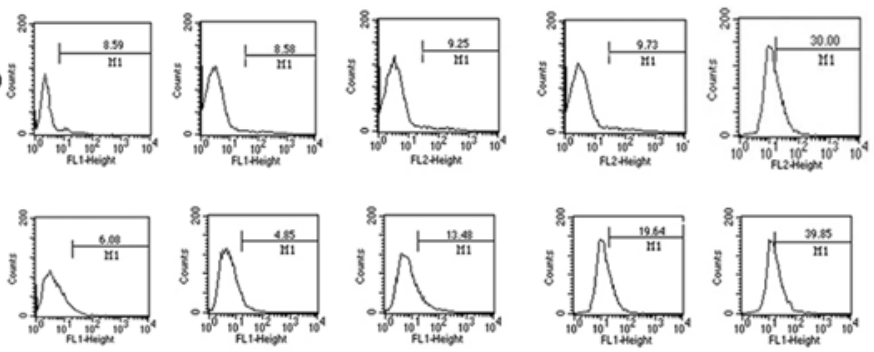

B
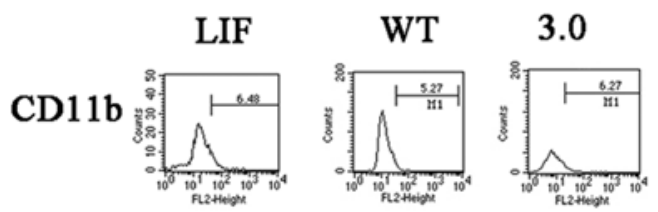

\section{MUT}

CT3
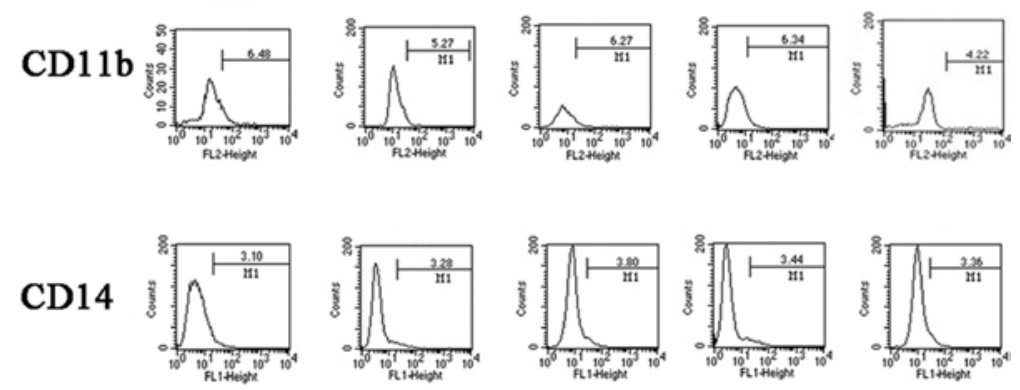

C

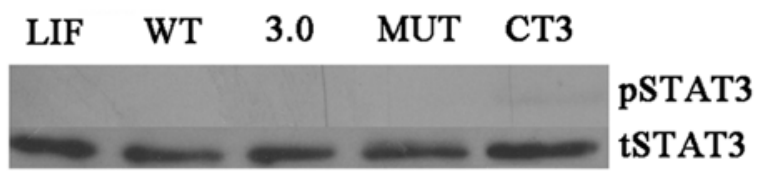

D

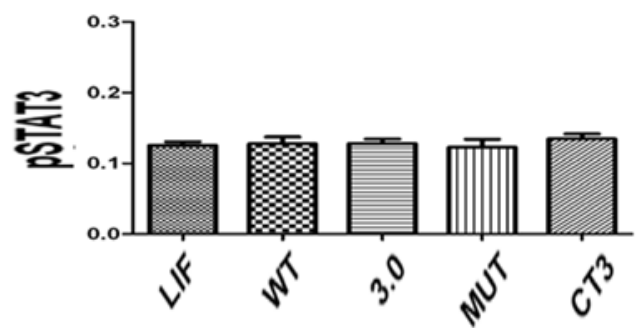

Figure 4. LIFR $\alpha$-CT3-induced up-regulation of pSTAT3 promotes differentiation of HL-60 cells. (A and B) Flow cytometry analysis of myeloid differentiation markers, CD11b and CD14, before and after addition of the STAT3-phosphorylation-specific inhibitor (25 $\mu \mathrm{M})$ to serum-free medium for $8 \mathrm{~h}$ (19,32). For the LIF group, the STAT3-phosphorylation-specific inhibitor $(25 \mu \mathrm{M})$ was added to starving HL-60 cells in serum-free medium for $8 \mathrm{~h}$ and rinsed three times with ice-cold 1X PBS followed by treatment with hLIF (100 ng/ml) for $30 \mathrm{~min}$ prior to being processed. The data represent three independent experiments. (C and D) pSTAT3 Y705 levels in total cell lysates were confirmed by Western blot analysis and corresponding densitometry analysis. tSTAT3 was chosen as a loading control. Values are the means \pm SEM ( $n=3$ for each group). $p>0.05$ when compared with the other groups.

have shown that STAT3 and/or STAT5 may be ideal targets for anti-cancer drugs $(22,25,26)$. We have observed that the levels of pSTAT3 are relatively high in all leukemia cells $(9,22,27)$, which is consistent with previous reports. After adding a specific Jak2 inhibitor to the culture media, all groups except the CT3 group showed a significant decrease in pSTAT3 levels. This observation indicates that i) the involvement of Jak2 can lead to endogenous STAT3 phosphorylation in HL-60 cells and can be effectively inhibited by AG-490 and ii) enrichment of LIFR $\alpha$-CT3 can enhance phosphorylation of intracellular STAT3 and cannot be inhibited by a Jak2-specific inhibitor. These observations provide evidence of a novel Jak2-independent mode for triggering STAT3 activation in HL-60 cells.

Upon the discovery of LIF, the up-regulation of pSTAT3 was observed to result in the terminal differentiation of HL-60 cells (28). While STAT3 phosphorylation is generally considered a subsequent outcome of Jak2 phosphorylation, its indirect activation in the cytoplasm by a single mediator may still be possible (10). This fact prompted us to examine whether we could enhance the activation of the LIF-related pathway to increase the efficiency of leukemia cell differentiation while avoiding the significant side effects that accompany the direct administration of LIF to target cells (24). In our study, we found that the CT3 group, compared to the other groups, showed a greater tendency to differentiate along the myeloid lineage upon pSTAT3 up-regulation. Our follow-up experiments further confirmed that the active site for these changes is LIFR $\alpha$-CT3. Interestingly, after STAT3 phosphorylation was blocked by a pharmacological inhibitor, a slight decrease in CD11b and CD14 levels was observed, indicating a modest positive correlation between pSTAT3 and the differentiation tendency of HL-60 cells. 
In the present study, transfection was performed to overexpress LIFR $\alpha$-CT3 in HL-60 cells. This technique could be improved, or replaced, by more novel methods, to avoid liposome involvement in cases of human genomic modification (data not shown). Our findings clearly demonstrate the ability of free cytoplasmic LIFR $\alpha$-CT3 to differentiate HL-60 cells via STAT3 phosphorylation. Nevertheless, the structural demands of recruiting STAT3 for phosphorylation still remain largely unexplored.

In conclusion, this study provides evidence that the most distal motifs of the LIFR $\alpha$ C-terminus can induce myeloid differentiation of HL-60 leukemia cells by enhancing Jak2independent STAT3 phosphorylation. As all-trans retinoid acid (ATRA) is currently the most widely applied clinical prescription for acute myeloid leukemia (AML) and acute promyeloid leukemia (APL), in spite of the risk of inducing the ATRA syndrome (29), the in vitro experiments presented herein demonstrate a potential molecular therapy with a purpose similar to ATRA, inducing the differentiation of leukemia cells instead of eliminating them. The introduction of LIFR $\alpha$-CT3-related domains by genomic modification or by other methods such as oligopeptide transduction may provide a promising novel therapeutic strategy for future treatment of AML/APL.

\section{Acknowledgements}

This study was supported by grants from the Science and Technology Commission of Shanghai Municipality (No. 09431901100).

\section{References}

1. Tomida M, Yamamoto-Yamaguchi Y and Hozumi M: Purification of a factor inducing differentiation of mouse myeloid leukemic M1 cells from conditioned medium of mouse fibroblast L929 cells. J Biol Chem 259: 10978-10982, 1984

2. Gearing DP, Gough NM, King JA, et al: Molecular cloning and expression of cDNA encoding a murine myeloid leukaemia inhibitory factor (LIF). EMBO J 6: 3995-4002, 1987.

3. Zhang Y, Willson T, Metcalf D, et al: The box-1 region of the leukemia inhibitory factor receptor alpha-chain cytoplasmic domain is sufficient for hemopoietic cell proliferation and differentiation. J Biol Chem 273: 34370-34383, 1998.

4. Levy-Nissenbaum O, Sagi-Assif O, Raanani P, Avigdor A, Ben-Bassat I and Witz IP: Overexpression of the dual-specificity MAPK phosphatase PYST2 in acute leukemia. Cancer Lett 199: 185-192, 2003.

5. Metcalf D: The leukemia inhibitory factor (LIF). Int J Cell Cloning 9: 95-108, 1991.

6. Hemmann U, Gerhartz C, Heesel B, et al: Differential activation of acute phase response factor/Stat 3 and Stat 1 via the cytoplasmic domain of the interleukin 6 signal transducer gp130. II. Src homology SH2 domains define the specificity of stat factor activation. J Biol Chem 271: 12999-13007, 1996.

7. Heim MH, Kerr IM, Stark GR and Darnell JE Jr: Contribution of STAT SH2 groups to specific interferon signaling by the Jak-STAT pathway. Science 267: 1347-1349, 1995.

8. Zhang T, Seow KT, Ong CT and Cao X: Interdomain interaction of Stat 3 regulates its Src homology 2 domain-mediated receptor binding activity. J Biol Chem 277: 17556-17563, 2002.

9. Liu H, Dan J, Tang S and Wu S: Involving of the cytoplasmic region of leukemia inhibitory factor receptor alpha subunit, IL-6 related signal transducer-gp130 or fas death domain for MAPK p42/44 activation in HL-60 cell with LIF or anti-Fas IgG. Mol Cell Biochem 217: 113-120, 2001.

10. White UA and Stephens JM: Neuropoietin activates STAT3 independent of LIFR activation in adipocytes. Biochem Biophys Res Commun 395: 48-50, 2010.
11. Moon DO, Kim MO, Lee JD, Choi YH and Kim GY: Butein suppresses c-Myc-dependent transcription and Akt-dependent phosphorylation of hTERT in human leukemia cells. Cancer Lett 286: 172-179, 2009.

12. Fang ZH, Dong CL, Chen Z, et al: Transcriptional regulation of survivin by c-Myc in BCR/ABL-transformed cells: implications in antileukemic strategy. J Cell Mol Med 13: 2039-2052, 2009.

13. Liu H, Liu S, Tang S, Ji K, Wang F and Hu S: Molecular analysis of signaling events mediated by the cytoplasmic domain of leukemia inhibitory factor receptor alpha subunit. Mol Cell Biochem 258: 15-23, 2004.

14. Shen ZX, Chen GQ, Ni JH, et al: Use of arsenic trioxide (As2O3) in the treatment of acute promyelocytic leukemia (APL): II. Clinical efficacy and pharmacokinetics in relapsed patients. Blood 89: 3354-3360, 1997.

15. Bach JP, Borta H, Ackermann W, Faust F, Borchers O and Schrader M: The secretory granule protein syncollin localizes to HL-60 cells and neutrophils. J Histochem Cytochem 54: $877-888,2006$

16. Bromberg JF, Wrzeszczynska MH, Devgan G, et al: Stat3 as an oncogene. Cell 98: 295-303, 1999.

17. Maekawa T and Metcalf D: Clonal suppression of HL60 and U937 cells by recombinant human leukemia inhibitory factor in combination with GM-CSF or G-CSF. Leukemia 3: 270-276, 1989.

18. Meydan N, Grunberger T, Dadi H, et al: Inhibition of acute lymphoblastic leukaemia by a Jak-2 inhibitor. Nature 379: 645-648, 1996

19. Giannopoulou M, Iszkula SC, Dai C, et al: Distinctive role of Stat 3 and Erk-1/2 activation in mediating interferon-gamma inhibition of TGF-betal action. Am J Physiol Renal Physiol 290: F1234-F1240, 2006

20. Quaglino A, Schere-Levy C, Romorini L, Meiss RP and Kordon EC: Mouse mammary tumors display Stat3 activation dependent on leukemia inhibitory factor signaling. Breast Cancer Res 9: R69, 2007.

21. Bitard J, Daburon S, Duplomb L, et al: Mutations in the immunoglobulin-like domain of gp190, the leukemia inhibitory factor (LIF) receptor, increase or decrease its affinity for LIF. J Biol Chem 278: 16253-16261, 2003.

22. Schuringa JJ, Wierenga AT, Kruijer W and Vellenga E: Constitutive Stat3, Tyr705, and Ser727 phosphorylation in acute myeloid leukemia cells caused by the autocrine secretion of interleukin-6. Blood 95: 3765-3770, 2000.

23. Tomida M, Heike T and Yokota T: Cytoplasmic domains of the leukemia inhibitory factor receptor required for STAT3 activation, differentiation, and growth arrest of myeloid leukemic cells. Blood 93: 1934-1941, 1999.

24. Metcalf D, Nicola NA and Gearing DP: Effects of injected leukemia inhibitory factor on hematopoietic and other tissues in mice. Blood 76: 50-56, 1990.

25. Obana M, Maeda M, Takeda K, et al: Therapeutic activation of signal transducer and activator of transcription 3 by interleukin-11 ameliorates cardiac fibrosis after myocardial infarction. Circulation 121: 684-691, 2010.

26. Buettner R, Mora LB and Jove R: Activated STAT signaling in human tumors provides novel molecular targets for therapeutic intervention. Clin Cancer Res 8: 945-954, 2002.

27. Emery B, Cate HS, Marriott M, et al: Suppressor of cytokine signaling 3 limits protection of leukemia inhibitory factor receptor signaling against central demyelination. Proc Natl Acad Sci USA 103: 7859-7864, 2006.

28. Nakajima K, Yamanaka Y, Nakae K, et al: A central role for Stat3 in IL-6-induced regulation of growth and differentiation in M1 leukemia cells. EMBO J 15: 3651-3658, 1996.

29. Tallman MS, Andersen JW, Schiffer CA, et al: All-trans-retinoic acid in acute promyelocytic leukemia. N Engl J Med 337: 1021-1028, 1997.

30. Chang KT, Tsai CM, Chiou YC, Chiu CH, Jeng KS and Huang CY: IL-6 induces neuroendocrine dedifferentiation and cell proliferation in non-small cell lung cancer cells. Am J Physiol Lung Cell Mol Physiol 289: L446-L453, 2005.

31. Levitzki A: Tyrphostins - potential antiproliferative agents and novel molecular tools. Biochem Pharmacol 40: 913-918, 1990.

32. Turkson J, Ryan D, Kim JS, et al: Phosphotyrosyl peptides block Stat3-mediated DNA binding activity, gene regulation, and cell transformation. J Biol Chem 276: 45443-45455, 2001. 